\title{
Computing control Lyapunov functions via a Zubov type algorithm $^{1}$
}

\author{
Lars Grüne \\ Fachbereich Mathematik \\ J.W. Goethe-Universität \\ 60054 Frankfurt a.M., Germany \\ gruene@math. uni-frankfurt.de
}

\author{
Fabian Wirth ${ }^{2}$ \\ Center for Technomathematics \\ University of Bremen \\ 28334 Bremen, Germany \\ fabian@math.uni-bremen.de
}

\begin{abstract}
We present a scheme for the determination of control Lyapunov functions which can be used as a basis for numerical computations. Under the assumption of local asymptotic nullcontrollability we define the domain of asymptotic nullcontrollability. On this set a control Lyapunov function is defined via an optimal control problem. It is then shown that this function can be characterized as the unique viscosity solution of a partial differential equation which can be interpreted as a generalization of Zubov's equation.
\end{abstract}

\section{Introduction}

Control Lyapunov functions have been shown to be an interesting tool in the analysis of nonlinear control systems. The existence of such a function can according to its regularity guarantee several interesting properties. The existence of a continuous control Lyapunov functions is equivalent to asymptotic nullcontrollability $[7,10]$. The existence of a continuously differentiable one, is equivalent to the existence of (possibly discontinuous) controllers robust with respect to measurement noise, [6]. Several design procedures are available that construct controllers given the knowledge of a control Lyapunov function. We refer to [9] for a good introduction to the area.

The use of control Lyapunov functions as a design tool is by now discussed in many textbooks, references can be found in [9]. Here, usually, design procedures are discussed that use a certain structure of the system in order to find control Lyapunov functions. A general procedure for their determination is not available. It is therefore of interest to turn to numerical methods for the approximation of control Lyapunov functions. In

\footnotetext{
${ }^{1}$ Research supported by the TMR Networks "Nonlinear Control Network" and "Viscosity Solutions and their applications" and DFG Priority Research Program "Ergodentheorie, Analysis und effiziente Simulation dynamischer Systeme"

${ }^{2}$ This paper was written while the author was a guest at the Centre Automatique et Systèmes, Ecole des Mines de Paris, Fontainebleau, France. The hospitality ot the members of the CAS is gratefully acknowledged.
}

this paper we present an approach to this end that is based on a generalization of a result by Zubov.

One of the celebrated results in the theory of ordinary differential equations is Zubov's method [12] which asserts that the domain of attraction of an asymptotically stable fixed point $x^{*}$ of

$$
\dot{x}=f(x), \quad x \in \mathbb{R}^{n}
$$

may be characterized by solutions $v$ of the partial differential equation

$$
D v(x) \cdot f(x)=-h(x)(1-v(x)) \sqrt{1+\|f(x)\|^{2}} .
$$

Namely, under suitable assumptions on $h$ the domain of attraction is the set $v^{-1}([0,1))$. The $v$ constructed via this equation is automatically a Lyapunov function and smooth. This result has recently be extended to perturbed systems in [2]. Numerical treatment of this equation is discussed in [3].

As we already know that it is unreasonable to expect continuously differentiable control Lyapunov functions, we will look for solutions of a generalization of (1) in the viscosity sense. We refer to [4] for an introduction to the theory and its close link to problems in optimal control. Given this connection it should come as no surprise that our approach is also heavily based on optimal control methods. In fact our procedure can be viewed as an extension to [7] where the equivalence of asymptotic nullcontrollability and the existence of a control Lyapunov function has been proved using basically the same idea. Here we take this approach a step further and obtain a characterization of control Lyapunov functions as unique viscosity solutions of a suitable PDE. In the following Section 2 we introduce the problem, define the domain of asymptotic controllability and show some first properties of it. The ensuing Section 3 is devoted to the proof that a certain class of control Lyapunov functions is characterized as a viscosity solution of a partial differential equation. We discuss some open problems in the concluding Section 4. 


\section{The domain of asymptotic null-controllability}

We consider a control system of the form

$$
\left\{\begin{array}{l}
\dot{x}(t)=f(x(t), u(t)), \quad t \in[0, \infty), \\
x(0)=x_{0},
\end{array}\right.
$$

where $u(\cdot) \in \mathcal{U}=L^{\infty}([0,+\infty), U)$ and $U$ is a compact subset of $\mathbb{R}^{m}, f$ is continuous and bounded in $\mathbb{R}^{n} \times U$ and Lipschitz in $x$ with Lipschitz constant independent of $u \in U$. Solutions of this system are denoted by $x\left(t, x_{0}, u\right)$. We will assume that $0 \in U$ and that $x=0$ is a fixed point under the control $u=0$, that is, $f(0,0)=0$.

We assume that the point 0 is uniformly locally asymptotically nullcontrollable (ULAM) for the system (2), i.e. there exist a constant $r>0$ and a function $\beta$ of class $\mathcal{K} \mathcal{L}^{1}$ such that for any $x_{0} \in B(0, r)$ there exists a $u \in \mathcal{U}$ such that $\left\|x\left(t, x_{0}, u\right)\right\| \leq \beta\left(\left\|x_{0}\right\|, t\right)$. It is known [8] that for any $\beta \in \mathcal{K} \mathcal{L}$ there exist two functions $\alpha_{1}, \alpha_{2} \in \mathcal{K}^{\infty}$ such that $\beta(r, t) \leq \alpha_{2}\left(\alpha_{1}(r) e^{-t}\right)$. For ease of presentation we will work with these two functions from now on.

The following definition specifies the set of initial conditions that may be asymptotically steered to zero.

Definition 2.1 Assume that (2) is ULAM. The domain of asymptotic nullcontrollability is defined by

$$
\mathcal{D}=\left\{x_{0} \in \mathbb{R}^{n} \mid \exists u \in \mathcal{U}: x\left(t, x_{0}, u\right) \rightarrow 0 \text { as } t \rightarrow+\infty\right\} .
$$

Let $\mathcal{D} \subset \mathbb{R}^{n}$ be the domain of asymptotic nullcontrollability of (2). A function $V: \mathbb{R}^{n} \rightarrow \mathbb{R}$ satisfying $V(0)=0, V(x)>0, x \neq 0$ is called a control Lyapunov function on $\mathcal{D}$, if it is proper on $\mathcal{D}$ and there exists a positive definite function $W: \mathbb{R}^{n} \rightarrow \mathbb{R}_{\geq 0}$ with

$$
\max _{\xi \in \partial_{P} V(x)} \min _{u \in U} \xi \cdot f(x, u) \leq-W(x), \forall x \in \mathcal{D}
$$

where $\partial_{P} V(x)$ denotes the proximal subgradient of $V$ in $x$, that is, the set of vectors $\xi \in \mathbb{R}^{n}$ such that there exists $\varepsilon, \sigma>0$ with

$V(y) \geq V(x)+\xi \cdot(y-x)-\sigma^{2}\|y-x\|^{2}, \forall\|x-y\|<\varepsilon$.

In order to obtain a different characterization of $\mathcal{D}$ we introduce the following "first hitting time" defined by $t(x, u):=\inf \{T>0: x(t, x, u) \in B(0, r)$ for all $t \geq$ $T\}$, where we set $\inf \emptyset=\infty$.

Lemma 2.2 Assume that (2) is ULAM, then

$$
\mathcal{D}=\left\{x \in \mathbb{R}^{n}: \inf _{u \in \mathcal{U}} t(x, u)<+\infty\right\} .
$$

Proof: This is immediate from Definition 2.1.

\footnotetext{
${ }^{1}$ As usual we call a function $\alpha$ of class $\mathcal{K}^{\infty}$ if it is a homeomorphism of $[0, \infty)$, a continuous function $\beta$ in two real nonnegative arguments is called of class $\mathcal{K} \mathcal{L}$ if it is of class $\mathcal{K}^{\infty}$ in the first and decreasing to zero in the second argument.
}

In the following proposition we present some relevant properties of the set $\mathcal{D}$. It will frequently be convenient to consider the reachable set at time $T$ from an initial condition $x_{0} \in \mathbb{R}^{n}$ defined by

$$
\mathcal{R}\left(x_{0}, T\right):=\left\{x \in \mathbb{R}^{n} \mid \exists u \in \mathcal{U} \text { so that } x=x\left(T, x_{0}, u\right)\right\} .
$$

Recall that a set $M$ is called weakly forward invariant for system (2) if for all $x \in M$ there exists a $u \in \mathcal{U}$ such that $x(t, x, u) \in M$ for all $t \geq 0$.

Proposition 2.3 Assume that (2) is ULAM, then

(i) $\operatorname{cl} B(0, r) \subset \mathcal{D}$.

(ii) $\mathcal{D}$ is an open, connected, weakly forward invariant set.

(iii) $\inf _{u \in \mathcal{U}}\{t(x, u)\} \rightarrow+\infty$ for $x \rightarrow x_{0} \in \partial \mathcal{D}$ or $\|x\| \rightarrow \infty$.

(iv) If for all $x \in \mathbb{R}^{n}$ the set $f(x, U)$ is convex then $\operatorname{cl} \mathcal{D}$ is weakly forward invariant.

Proof: (i) Assume that for some $x \in \partial B(0, r)$ we have $x \notin \mathcal{D}$. Let $\left\{x_{n}\right\} \subset B(0, r)$ be a sequence with $\lim _{n \rightarrow \infty} x_{n}=x$. By assumption to each $x_{n}$ there exists a control $u_{n} \in \mathcal{U}$ such that $\left\|x\left(t, x_{n}, u_{n}\right)\right\| \leq$ $\alpha_{2}\left(\alpha_{1}(r) e^{-t}\right)$. Choosing a subsequence we may assume that $x\left(\cdot, x_{n}, u_{n}\right) \rightarrow y(\cdot)$ uniformly on compact intervals. By [1, Theorem 2.2.1] it follows that $y$ is a solution of

$$
\dot{y} \in \operatorname{conv} f(y, U) \text {. }
$$

By construction $\|y(t)\| \leq \alpha_{2}\left(\alpha_{1}(r) e^{-t}\right)$, so that $y(t) \in$ $B(0, r / 2)$ for some $t$ large enough. Now by [1, Theorem 2.4.2] there is a sequence $x\left(\cdot, x, v_{n}\right)$ for some controls $v_{n} \in \mathcal{U}$ converging uniformly to $y$. It follows that $x\left(t, x, v_{n}\right) \in B(0, r)$ for some $n$ large enough which shows that $x$ is asymptotically null controllable.

(ii) Let $x_{0} \in \mathcal{D}$. Then there exist $T \in \mathbb{R}$ and $u \in \mathcal{U}$ such that $x\left(T, x_{0}, u\right) \in B(0, r)$. By continuous dependence on the initial value a neighborhood of $x_{0}$ is mapped into $B(0, r)$ under the map $y \mapsto x(T, y, u)$ and it follows that $\mathcal{D}$ is open.

Furthermore, by definition from each $x \in \mathcal{D}(x \in \mathcal{D})$ there exists a trajectory $x(\cdot, x, u)$ entering $B(0, r)$. This shows connectedness. Weak invariance is obvious from the definition.

(iii) Let $x_{n} \rightarrow x_{0} \in \partial \mathcal{D}$ and set $T_{n}=\inf _{u \in \mathcal{U}}\left\{t\left(x_{n}, u\right)\right\}$. If we assume that $\left\{T_{n}\right\}$ is bounded, we can find $T$ such that, for any $n$ there is a control $u_{n}$ such that $x\left(T, x_{n}, u_{n}\right) \in B(0, r)$. Now we can argue as in (i) to construct a solution from $x_{0}$ into $B(0, r)$ which contradicts the assumption that $x_{0} \notin \mathcal{D}$. The assertion is clear for $\left\|x_{n}\right\| \rightarrow \infty$, as our assumptions exclude solutions exploding in backward time. 
(iv) It is sufficient to show that for every $x \in \operatorname{cl} \mathcal{D}$ and all $t>0$ there exists a $u \in \mathcal{U}$ such that $x(t, x, u) \in \operatorname{cl} \mathcal{D}$. Then the claim follows by concatenation. Assume this is not the case for $x_{0} \in \operatorname{cl} \mathcal{D}$, then $\mathcal{R}\left(x_{0}, T\right) \cap \operatorname{cl} \mathcal{D}=\emptyset$. As $\mathcal{R}\left(x_{0}, T\right)$ is compact by [1, Theorem 2.2.1] it follows that there is a neighborhood $V$ of $\mathcal{R}\left(x_{0}, T\right)$ such that $V \cap \operatorname{cl} \mathcal{D}=\emptyset$. Now by continuous dependence on initial conditions a neighborhood of $x_{0}$ is mapped into $V$ by the map $x \mapsto x(T, \cdot, u)$ for any $u \in \mathcal{U}$. This contradicts weak invariance of $\mathcal{D}$.

\section{Zubov's method for domains of asymptotic null controllability}

It is our aim to show that some control Lyapunov functions may be characterized as viscosity solutions and that the set $\mathcal{D}$ may be characterized with the help of these functions. Before turning to this problem we introduce two optimal value functions and show certain properties of these functions. This will enable us to use standard techniques from the theory of HamiltonJacobi-Bellman equations in the proofs.

Consider the following nonnegative, extended value function $V: \mathbb{R}^{n} \rightarrow \mathbb{R} \cup\{+\infty\}$ given by the optimal control problem

$J(x, u):=\int_{0}^{+\infty} g(x(t), u(t)) d t, \quad V(x)=\inf _{u \in \mathcal{U}} J(x, u)$.

We will also consider its transformation via the Kruzkov transform

$$
v(x)=1-e^{-V(x)} .
$$

The function $g: \mathbb{R}^{n} \times U \rightarrow \mathbb{R}_{\geq 0}$ is assumed to be continuous and satisfies furthermore

(A i) If $x \in B(0, r)$ then $\|g(x, u)\| \leq C \alpha_{2}^{-1}(\|x\|)$, $g(x, u)=0$, iff $x=0$

(A ii) There exists a constant $g_{0}>0$ such that $\inf _{x \notin B(0, r), u \in U} g(x, u) \geq g_{0}$.

(A iii) For every $R>0$ there exists a constant $L_{R}$ such that $\|g(x, u)-g(y, u)\| \leq L_{R}\|x-y\|$ for all $\|x\|$, $\|y\| \leq R$, and all $u \in U$.

Since $g$ is nonnegative it is immediate that $V(x) \geq 0$ and $v(x) \in[0,1]$ for all $x \in \mathbb{R}^{n}$. Furthermore, standard techniques from optimal control (see e.g. [4, Chapter III]) imply that $V$ and $v$ satisfy the dynamic programming principle, i.e. for each $t>0$ we have

$$
V(x)=\inf _{u \in \mathcal{U}}\left\{\int_{0}^{t} g(x(\tau, x, u), u(\tau)) d \tau+V(x(t, x, u))\right\}
$$

and

$$
v(x)=\inf _{u \in \mathcal{U}}\{(1-G(x, t, u))+G(x, t, u) v(x(t, x, u))\}
$$

with

$$
G(x, t, u):=\exp \left(-\int_{0}^{t} g(x(\tau, x, u), u(\tau)) d \tau\right) .
$$

In the next proposition we investigate the relation between $\mathcal{D}$ and $V$ (and thus also $v$ ), and the continuity of $V$ and $v$.

Proposition 3.1 Assume that (2) is ULAM and that $g$ satisfies (A i) - (A iii). Then

(i) $V(x)<+\infty$ if and only if $v(x)<1$ if and only if $x \in \mathcal{D}$.

(ii) $V(0)=0$ if and only if $v(0)=0$ if and only if $x=0$.

(iii) $V$ is continuous on $\mathcal{D}, v$ is continuous on $\mathbb{R}^{n}$.

(iv) $V(x) \rightarrow+\infty$ and $v(x) \rightarrow 1$ for $x \rightarrow x_{0} \in \partial \mathcal{D}$ and for $\|x\| \rightarrow \infty$.

Proof: (i) To show that $V\left(x_{0}\right)<+\infty$ for $x_{0} \in \mathcal{D}$, observe that by Lemma 2.2 for each $x_{0} \in \mathcal{D}$ there exist $T_{0}>0, u \in \mathcal{U}$ such that $x\left(t, x_{0}, u\right) \in B(0, r)$ for all $t \geq T_{0}$. Thus $J\left(x_{0}, u\right)$ is bounded by

$$
\begin{gathered}
\int_{0}^{T_{0}} g(x(t), u(t)) d t+C \int_{T_{0}}^{+\infty} \alpha_{1}\left(\left\|x\left(T_{0}\right)\right\|\right) e^{T_{0}-t} d t \\
=\int_{0}^{T_{0}} g(x(t), u(t)) d t+C \alpha_{1}\left(\left\|x\left(T_{0}\right)\right\|\right)<\infty .
\end{gathered}
$$

and therefore $V\left(x_{0}\right)<+\infty$.

Now let $x_{0} \notin \mathcal{D}$. Then for any $u \in \mathcal{U}$ we have $g\left(x\left(t, x_{0}, u\right), u(t)\right) \geq g_{0}$, where $g_{0}>0$ is defined as in (A ii). It follows that $J(x, u)=\infty$ and hence $V(x)=+\infty$. Now the assertion for $v$ is immediate.

(ii) This follows immediately from (3), (A i), and $f(0,0)=0$.

(iii) Fix $x_{0} \in B\left(0, \alpha_{1}^{-1}\left(\alpha_{2}^{-1}(r)\right)\right)$. Then local asymptotic nullcontrollability and (A i) imply that for some $u_{0} \in \mathcal{U}$ we have

$$
\begin{array}{r}
V\left(x_{0}\right) \leq \int_{0}^{+\infty} g\left(x\left(t, x_{0}, u_{0}\right), u_{0}(t)\right) d t \\
\leq C \int_{0}^{+\infty} \alpha_{2}^{-1}\left(\left\|x\left(t, x_{0}, u_{0}\right)\right\|\right) d t \\
\leq C \int_{0}^{+\infty} e^{-t} \alpha_{1}\left(\left\|x_{0}\right\|\right) d t \leq C \alpha_{1}\left(\left\|x_{0}\right\|\right) .
\end{array}
$$


Now fix $\varepsilon>0$ and $r^{*}<\alpha_{1}^{-1}\left(\alpha_{2}^{-1}(r)\right) \leq r$ such that $C \alpha_{1}\left(r^{*}\right)<\varepsilon$. Let $x \in \mathcal{D}$ be arbitrary. By assumption there are $u \in \mathcal{U}$ and $T>0$ such that $\|x(T, x, u)\| \leq r^{*} / 2$ and $V(x)+\varepsilon>J(x, u)$. By continuous dependence on the initial value there is a neighborhood $W \subset \mathcal{D}$ of $x$ such that $\|x(T, y, u)\| \leq r^{*}$ for all $y \in W$. We may assume that $\operatorname{cl} W \subset \mathcal{D}$ is compact, whence also $\mathcal{R}:=\mathrm{cl} \cup_{x \in W, t \in\left[0, T^{*}\right]} \mathcal{R}(x, t)$ is compact. This implies in particular, that $V(y) \leq T \max \{g(x, u) \mid x \in \mathcal{R}, u \in$ $U\}+\varepsilon$ for all $y \in W$. From the boundedness of $V$ on $W$ and the fact that $g$ is bounded away from zero on $\mathbb{R}^{n} \backslash B\left(0, r^{*} / 2\right)$ it follows that there is a $T^{*}$ such that whenever $y \in W$ and $V(y)+\varepsilon>J(y, u)$ we have $x(t, y, u) \in B\left(0, r^{*} / 2\right)$ for all $t>T^{*}$. We may now choose Lipschitz constants $L_{f}, L_{g}$ for $f$ and $g$ on $\mathcal{R}$, respectively. Let $y, z \in W$ and $u$ be such that $V(y)+\varepsilon>$ $J(y, u)$. Then we obtain

$$
\begin{array}{r}
V(z)-V(y)<V(z)-\int_{0}^{+\infty} g(x(t, y, u), u(t)) d t+\varepsilon \\
\leq \int_{0}^{T^{*}}|g(x(t, z, u), u(t))-g(x(t, y, u), u(t))| d t+ \\
V\left(x\left(T^{*}, z, u\right)\right)+C \alpha_{1}\left(r^{*}\right)+\varepsilon \\
\leq L_{g} \int_{0}^{T^{*}} e^{L_{f} t}\|z-y\| d t+V\left(x\left(T^{*}, z, u\right)\right)+2 \varepsilon
\end{array}
$$

If $\|z-y\|$ is small enough then $x\left(T^{*}, z, u\right) \in B\left(0, r^{*}\right)$ so that $V\left(x\left(T^{*}, z, u\right)\right)<C \alpha_{1}\left(r^{*}\right)$ and also the integral can be bounded by $\varepsilon$, so that the whole expression is bounded by $4 \varepsilon$. As this condition is symmetric in $y, z$ this shows continuity of $V$. The function $v$ is then continuous by definition.

(iv) The statement follows immediately from Proposition 2.3 (iii) since $\mathcal{D}$ is open and $g(x, u) \geq g_{0}>0$ for $x$ outside of $\mathrm{cl} B(0, r)$ as assumed in (A ii).

We now turn to the formulation of suitable partial differential equations for which $V$ and $v$ form solutions. Since in general these functions will not be differentiable we have to work with a more general solution concept, namely viscosity solutions.

Let us recall the definition of viscosity solutions (for more details about this theory we refer to [4]).

Definition 3.2 Given an open subset $\mathcal{O}$ of $\mathbb{R}^{n}$ and a continuous function $H: \mathcal{O} \times \mathbb{R} \times \mathbb{R}^{n} \rightarrow \mathbb{R}$, we say that a lower semicontinuous (l.s.c.) function $u: \mathcal{O} \rightarrow \mathbb{R}$ (resp. an upper semicontinuous (u.s.c.) function $v: \mathcal{O} \rightarrow \mathbb{R}$ ) is a viscosity supersolution (resp. subsolution) of the equation

$$
H(x, u, D u)=0 \quad x \in \mathcal{O}
$$

if for all $\phi \in C^{1}(\mathcal{O})$ and $x \in \operatorname{argmin}_{\mathcal{O}}(u-\phi$ ) (resp., $\left.x \in \operatorname{argmax}_{\mathcal{O}}(v-\phi)\right)$ we have

$$
H(x, u(x), D \phi(x)) \geq 0(\text { resp., } H(x, v(x), D \phi(x)) \leq 0) \text {. }
$$

A continuous function $u: \mathcal{O} \rightarrow \mathbb{R}$ is said to be a viscosity solution of (8) if $u$ is a viscosity supersolution and a viscosity subsolution of (8).

Recalling that $V$ is locally bounded in $\mathcal{D}$, and $v$ is locally bounded on $\mathbb{R}^{n}$ the following proposition follows from an easy application of the dynamic programming principles (5) and (6), cp. [4, Chapter III].

Proposition 3.3 $V$ is a viscosity solution of

$$
\sup _{u \in U}\{-D V(x) f(x, u)-g(x, u)\}=0, \quad x \in \mathcal{D}
$$

and $v$ is a viscosity solution of

$$
\sup _{u \in U}\{-D v(x) f(x, u)-(1-v(x)) g(x, u)\}=0, x \in \mathbb{R}^{n} .
$$

Observe that (10) is the straightforward generalization of the classical Zubov equation (1) introduced in [12]. Also our "auxiliary function" $V$ can be characterized as the solution of a suitable PDE. It might be considered to be more natural to write $\inf _{u \in U}\{D v(x) f(x, u)+(1-v(x)) g(x, u)\}=0$. However, this would be in disaccord with the standard way of defining sub- and supersolutions. We note the following immediate consequence.

Corollary 3.4 The functions $V, v$ are control Lyapunov functions on $\mathcal{D}$.

Proof: The equations (9) and (10) incorporate already the definition of the function $W$ that is required in the definition of control Lyapunov functions. Properness on $\mathcal{D}$ follows from Proposition 2.3 (iv). The condition on the proximal subgradients follows from the properties of supersolutions.

In order to prove uniqueness of the solutions we need the following optimality principles. The statement is an immediate consequence of [11, Theorem 3.2 (i) \& (iii)].

\section{Proposition 3.5}

(i) Let $w$ be a u.s.c. subsolution of (10) in $\mathbb{R}^{n}$, then for any $x \in \mathbb{R}^{n}$

$$
w(x)=\inf _{u \in \mathcal{U}} \inf _{t \geq 0}\{1+G(x, t, u)(w(x(t))-1)\} .
$$

(ii) Let $W$ be a u.s.c. subsolution of (10) in $\mathcal{D}$, then for any $x \in \mathcal{D}$

$$
W(x)=\inf _{u \in \mathcal{U}} \inf _{t \geq 0}\left\{\int_{0}^{t} g(x(s), u(s)) d s+W(x(t))\right\} .
$$


(iii) Let $w$ be a l.s.c. supersolution of (10) in $\mathbb{R}^{n}$, then for any $x \in \mathbb{R}^{n}$

$$
w(x) \geq \inf _{u \in \mathcal{U}} \sup _{t \geq 0}\{1+G(x, t, u)(w(x(t))-1)\} .
$$

We can now apply these principles to the generalized version of Zubov's equation (10).

Proposition 3.6 Let $w$ be a bounded l.s.c. supersolution of $(10)$ on $\mathbb{R}^{n}$ with $w(0) \geq 0$. Then $w \geq v$ for $v$ as defined in (4).

Proof: Let $M>0$ be a bound on $|w|$. If for some control $u$ and some $\eta>0$ it holds that $\left\|x\left(t, x_{0}, u\right)\right\| \geq \eta$ for all $t \geq 0$ then it follows from assumption (H1) that $G\left(x_{0}, t, u\right) \leq \exp \left(-t g_{\eta}\right)$ for a suitable constant $g_{\eta}$. This implies

$$
\begin{gathered}
\sup _{t \geq 0}\left\{1+G\left(x_{0}, t, u\right)(w(x(t))-1)\right\} \geq \\
\sup _{t \geq 0}\left\{\left(1-\exp \left(-t g_{\eta}\right)\right)-\exp \left(-t g_{\eta}\right) M\right\}=1 .
\end{gathered}
$$

Hence, if for some $x_{0} \in \mathbb{R}^{n}$ the infimum in (13) is approximated via trajectories that are bounded away from the origin, then $w\left(x_{0}\right) \geq 1$. In particular, this is the case if $x_{0} \notin \mathcal{D}$ by the forward invariance of $\mathcal{D}^{c}$. Hence using Proposition 3.1 (i), we have $w\left(x_{0}\right) \geq 1=v\left(x_{0}\right)$ for $x_{0} \in \mathcal{D}^{c}$. If $x_{0} \in \mathcal{D}$ and $w\left(x_{0}\right) \geq 1$, then again Proposition 3.1 (i) implies the assertion, so that it remains to show the claim under the conditions $x_{0} \in \mathcal{D}$ and $w\left(x_{0}\right)<1$. Fix $\left(1-w\left(x_{0}\right)\right) / 2>\varepsilon>0$ and choose $u_{\varepsilon}$ such that

$$
\begin{gathered}
\sup _{t \geq 0}\left\{1+G\left(x_{0}, t, u_{\varepsilon}\right)(w(x(t))-1)\right\}-\varepsilon< \\
\inf _{u \in \mathcal{U}} \sup _{t \geq 0}\left\{1+G\left(x_{0}, t, u\right)(w(x(t))-1)\right\} .
\end{gathered}
$$

This implies in particular, that $x\left(t, x_{0}, u_{\varepsilon}\right)$ is not bounded away from the origin. Now observe that the lower semicontinuity of $w$ and the assumption $w(0) \geq 0$ imply that there exists a $\delta>0$ such that

$$
w(x) \geq-\varepsilon \text { for all }\|x\| \leq \delta .
$$

Hence there exists a sequence $t_{n} \rightarrow \infty$ such that for all $n \in \mathbb{N}$ we have $w\left(x\left(t_{n}, x_{0}, u_{\varepsilon}\right)\right) \geq-\varepsilon$ and

$$
\left|G\left(x_{0}, t_{n}, u_{\varepsilon}\right)-G\left(x_{0}, \infty, u_{\varepsilon}\right)\right| \leq \varepsilon .
$$

Thus from (14) and (13), and using the definition of $v$ we can conclude

$$
\begin{aligned}
& w\left(x_{0}\right) \geq \limsup _{n \rightarrow \infty}\left\{1+G\left(x_{0}, t_{n}, u_{\varepsilon}\right)\left(w\left(x\left(t_{n}\right)\right)-1\right)\right\}-\varepsilon \\
& \geq v\left(x_{0}\right)-\varepsilon\left(G\left(x_{0}, \infty, u_{\varepsilon}\right)+\varepsilon+1\right) \geq v\left(x_{0}\right)-\varepsilon(2+\varepsilon),
\end{aligned}
$$

which shows the claim, as $\varepsilon>0$ is arbitrary.
Proposition 3.7 Let $w$ be a bounded u.s.c. subsolution of $(10)$ on $\mathbb{R}^{n}$ with $w(0) \leq 0$. Then $w \leq v$ for $v$ defined in (4).

Proof: $\quad$ By the upper semicontinuity of $w$ and $w(0) \leq$ 0 we obtain that for every $\varepsilon>0$ there exists a $\delta>0$ with $w(x) \leq \varepsilon$ for all $x \in \mathbb{R}^{n}$ with $\|x\| \leq \delta$. Now we distinguish two cases:

(i) $x_{0} \in \mathcal{D}:$ We choose $u^{*} \in \mathcal{U}$ such that $v\left(x_{0}\right)+\varepsilon>1-$ $G\left(x_{0}, \infty, u^{*}\right)$. In particular, this implies $x\left(t, x_{0}, u^{*}\right) \rightarrow$ 0 as $t \rightarrow \infty$. Thus it follows from the lower optimality principle (11) and the definition of $v$ that

$$
\begin{gathered}
w\left(x_{0}\right) \leq \limsup _{t \rightarrow \infty} 1+G\left(x_{0}, t, u^{*}\right)\left(w\left(x\left(t, x_{0}, u^{*}\right)\right)-1\right) \\
\leq 1+G\left(x_{0}, \infty, u^{*}\right)(\varepsilon-1) \leq v\left(x_{0}\right)+2 \varepsilon
\end{gathered}
$$

which shows the claim as $\varepsilon>0$ was arbitrary.

(ii) $x_{0} \notin \mathcal{D}$ : In this case by Proposition 3.1 (i) it is sufficient to show that $w\left(x_{0}\right) \leq 1$. Let $M$ be a bound on $|w|$. Since $x\left(t, x_{0}, u\right) \notin \mathcal{D}$ for all $u \in \mathcal{U}$ we have $G\left(x_{0}, t, u\right) \leq \exp \left(-g_{0} t_{n}\right)$ for all $t \geq 0, u \in \mathcal{U}$. Hence

$$
\begin{gathered}
1+G\left(x_{0}, t, u\right)\left(w\left(x\left(t, x_{0}, u\right)\right)-1\right) \\
\leq 1+\exp \left(-g_{0} t\right)(M+1)
\end{gathered}
$$

and the result follows by (11) as the right hand side tends to 1 for $t \rightarrow \infty$.

Using these propositions we can now formulate an existence and uniqueness theorem for the generalized version of Zubov's equation (10).

Theorem 3.8 Consider the system (2) and a function $g: \mathbb{R}^{n} \times U \rightarrow \mathbb{R}$ such that (H1) and (H2) are satisfied. Then (10) has a unique bounded and continuous viscosity solution $v$ on $\mathbb{R}^{n}$ satisfying $v(0)=0$.

This function coincides with $v$ from (4). In particular the characterization $\mathcal{D}=\left\{x \in \mathbb{R}^{n} \mid v(x)<1\right\}$ holds.

Proof: This is immediate from Propositions 3.6 and 3.7 .

For the sake of completeness we state the analogous result for equation (9) which is proved with the same techniques, using the obvious modifications of (11) and (13). Observe that this result corresponds to the one in $[5]$.

Theorem 3.9 Consider the system (2) and a function $g: \mathbb{R}^{n} \times U \rightarrow \mathbb{R}$. Assume (H1) and (H2). Let $\mathcal{O} \subset \mathbb{R}^{n}$ be an open set containing the origin, and let $P: \mathcal{O} \rightarrow \mathbb{R}$ be 
a positive and continuous function which is a viscosity solution of (9) on $\mathcal{O}$ and satisfies $P(0)=0$ and $P(x) \rightarrow$ $\infty$ for $x \rightarrow \partial \mathcal{O}$ and for $|x| \rightarrow \infty$.

Then $P$ coincides with $V$ from $(3)$ and $\mathcal{O}=\mathcal{D}$. In particular, the function $V$ from (3) is the unique positive continuous viscosity solution of equation (9) on $\mathcal{D}$ with $V(0)=0$.

As in [2] it can be shown that we can restrict ourselves to a proper open subset $\mathcal{O}$ of the state space and still obtain our solution $v$, provided $\mathcal{D} \subseteq \mathcal{O}$. We omit the discussion of this aspect, for reasons of space.

Finally, we return to the point that the function $v$ is a control Lyapunov function for the system (2). In particular each sublevel set of $v$ is weakly forward invariant. The following bound may be obtained for the amount of decrease that can be obtained in terms of $v$.

Theorem 3.10 For all $x_{0} \in \mathcal{D} \backslash\{0\}$ there exists a $u \in$ $\mathcal{U}$ such that

$$
\begin{gathered}
v\left(x\left(t, x_{0}, u\right)\right)-v\left(x_{0}\right) \leq \\
{\left[1-\exp \left(-\int_{0}^{t} g(x(\tau), u(\tau)) d \tau\right)\right]\left(v\left(x\left(t, x_{0}, u\right)\right)-1\right) .}
\end{gathered}
$$

Proof: $\quad$ By $(6)$ for each $x \neq 0$ there exists a $u \in \mathcal{U}$ such that $v(x)$ is bounded from below by

$$
\begin{gathered}
1-\exp \left(\int_{0}^{t} g(x(\tau, x, a), a(\tau)) d \tau\right)+ \\
\exp \left(\int_{0}^{\tau} g(x(\tau, x, a), a(\tau)) d \tau\right) v(x(t, x, u)) .
\end{gathered}
$$

This immediately yields the assertion.

\section{Conclusions}

This paper contains some first results on the relation of control Lyapunov functions and viscosity solutions, but the story seems to be far from complete. The experienced reader will have noticed that we avoided any problems with the boundedness of control functions by assuming that $U$ is compact. The standard definition allows for non-compact $U$ and requires in addition that on each compact subset of the state space it is sufficient to choose control values from a compact set in order to drive the system to zero. In terms of the Zubov equation this means that large values of $u$ should to be penalized. The construction then becomes a little more involved. That we also required $f$ to be bounded on the other hand is no big drawback, as one might always replace $f$ by $f /(1+\|f\|)$ without changing the systems trajectories. Though of course other points of interest in design are lost.

A further question is what subset of control Lyapunov functions may be realized as solutions to a Zubov equation by appropriate choice of $g$. This is the subject of ongoing research.

\section{References}

[1] J.-P. Aubin and A. Cellina. Differential Inclusions: Set-Valued Maps and Viability Theory. Springer-Verlag, 1984.

[2] F. Camilli, L. Grüne and F. Wirth. A generalization of Zubov's method to perturbed systems. SIAM J. Control Optim., 2000. to appear.

[3] F. Camilli, L. Grüne and F. Wirth. A regularization of Zubov's equation for robust domains of attraction. in Nonlinear Control in the Year 2000, A. Isidori et. al. eds., Lecture Notes in Control and Information Sciences, SpringerVerlag, London, 2000, to appear.

[4] M. Bardi and I. Capuzzo Dolcetta, Optimal Control and Viscosity Solutions of Hamilton-Jacobi-Bellman equations, Birkhäuser, Boston, 1997.

[5] M. Bardi and P. Soravia, Hamilton-Jacobi equations with singular boundary conditions on a free boundary and applications to differential games, Trans. Amer. Math. Soc. 325 (1991), 205-229.

[6] Yu.S. Ledyaev and E.D. Sontag. A Lyapunov characterization of robust stabilization. J. Nonlinear Analysis TMA 37 (1999), 813-840.

[7] E.D. Sontag. A Lyapunov-like characterization of asymptotic controllability, SIAM J. Control Optim., 21 (1983), 462-471.

[8] E.D. Sontag. Comments on integral variants of ISS, Syst. \& Contr. Lett., 34 (1998), 93-100.

[9] E.D. Sontag. Stability and stabilization: Discontinuities and the effect of disturbances. In: F.H. Clarke and R.J. Stern, eds. Nonlinear Analysis, Differential Equations, and Control (Proc. NATO Advanced Study Institute, Montreal, Jul/Aug 1998), Kluwer, 1999, pp. 551-598.

[10] E.D. Sontag and H.J. Sussmann. Nonsmooth controlLyapunov functions. In Proc. CDC 1995, New Orleans, USA, 1995, pp. 2799-2805.

[11] P. Soravia. Optimality principles and representation formulas for viscosity solutions of Hamilton-Jacobi equations, I: Equations of unbounded and degenerate control problems without uniqueness, Advances Diff. Equations, 4 (1999), 275-296.

[12] V.I. Zubov. Methods of A.M. Lyapunov and their Application, P. Noordhoff, Groningen, 1964. 